\title{
Symbolic comparisons with and without perceptual referents: Is interval information used?
}

\author{
JOHN M. HENDERSON and ARNOLD D. WELL \\ University of Massachusetts, Amherst, Massachusetts
}

\begin{abstract}
In an attempt to investigate the range effect obtained by Moyer and Bayer (1976), two groups of subjects were taught to associate colors with circles that differed in size by larger or smaller intervals and then participated in a symbolic comparison task, in which they determined which of two simultaneously presented symbols (the first letters of two colors) represented the larger magnitude. A third group of subjects, who merely learned an arbitrary ordering of the colors along the dimension of size, also participated in the symbolic comparison task. Despite the fact that no practice trials were given, there was no effect of interval size in either early or late blocks, and the circle-learning groups did not differ in performance from the group who only learned an arbitrary ordering of colors, even when pairs containing end terms were removed from the analysis. It is concluded that subjects do not use analogue codes in symbolic comparison tasks involving small, repeated sets of arbitrarily defined symbols.
\end{abstract}

Recently, much research (e.g., Banks, White, Sturgill, \& Mermelstein, 1983; Holyoak, Dumais, \& Moyer, 1979; Moyer, 1973; Paivio, 1975) has focused on symbolic comparison, ${ }^{1}$ in which the cognitive referents of two or more symbols are compared along some common dimension. For example, the cognitive referents of the words (i.e., symbols) "dog" and "cat" could be compared along the dimension of size. Symbolic comparison typically has been investigated by presenting subjects with pairs of $a b-$ stract symbols [either words or experimenter-defined symbols such as consonant-vowel-consonants (CVCs)] that have referents represented in memory, and recording the time taken to determine which referent is more extreme on the dimension in question. In some studies, the referents have been mental representations of positions on an arbitrary linear ordering (e.g., A < B < C; Potts, 1972, 1974), whereas in others, the referents have been mental representations of physical objects whose relationships along a given dimension are defined by their physical attributes. A purpose of the present experiment is to determine whether similar processes are used in these two types of symbolic comparison.

In previous studies, it has been found that "the time needed to compare two symbols varies inversely with the distance between their referents on the judged dimension" (Moyer \& Bayer, 1976, p. 230). This effect is generally called the "symbolic distance effect," and has been found in symbolic comparisons using diverse stimuli (e.g., McKinley, 1975; McKinley-Brewer, 1979; Moyer, 1973;

We gratefully acknowledge the assistance of Jim Chumbley, Nelson Cowan, Fernanda Ferreira, John Pani, and Sandy Pollatsek for comments on a draft of this article. Requests for reprints should be sent to John Henderson, Department of Psychology, University of Massachusetts, Amherst, MA 01003.
Paivio, 1975, 1978). The robust and ubiquitous nature of this effect is important because it constrains the types of models that can successully explain symbolic comparison. The explanations put forth for this effect to date (see Banks, 1977, Moyer \& Dumais, 1978, and Potts, Banks, Kosslyn, Moyer, Riley, \& Smith, 1978, for reviews) fall into two general classes.

The first general class of explanation may be termed the analogue representation hypothesis. As Moyer has pointed out, the response-time functions found in studies of symbolic comparison are similar to those found in studies of perceptual comparison (Potts et al., 1978). The similarity between symbolic and perceptual comparisons has led several investigators to postulate that both types of comparisons employ a common or functionally similar representation and/or comparison stage (e.g., Jamieson \& Petrusic, 1975; Moyer, 1973; Paivio, 1975; see also Finke, 1980, and Shepard \& Podgorny, 1978). On this view, the internal representation accessed from memory in a symbolic comparison maintains continuous metric information along the perceptual dimension. It has been suggested that such representations might serve as input to imagery-based (e.g., Kosslyn, Murphy, Bemesderfer, \& Feinstein, 1977; Moyer, 1973; Paivio, 1975) or stochastic sampling (e.g., Buckley \& Gillman, 1974; Marks, 1972) comparison processes.

The second general class of explanation may be termed the discrete representation hypothesis. According to this view, the nature of the code accessed from memory in a symbolic comparison is more abstract than an analogue code, retaining only information about relative position along the dimension of comparison. The clearest instantiation of this representational assumption is the semantic coding model advanced by Banks (1977; but see also Woocher, Glass, \& Holyoak, 1978), in which proposi- 
tional or linguistic codes indicating the relative position of each item along the dimension of comparison are compared by seeking a match between an accessed code (e.g., "large") and a similarly formatted coding of the instructions (e.g., "choose large"). In studies using large sets of semantically defined symbols such as animal names, it seems unlikely that linguistic codes indicating relative position along the comparison dimension would have been precomputed and stored in semantic memory (see Banks, 1977). However, when the pairs of symbols being compared originate from a finite set, especially a small finite set, it would seem reasonable to suppose that a representation of the relative ordering of the symbols could be constructed and held in working memory, so that analogue representations would be unnecessary.

However, Moyer and Bayer (1976) provided evidence for the analogue representation hypothesis and against the semantic coding model in a study in which only four arbitrarily ordered symbols with perceptual referents were repeatedly compared. Moyer and Bayer had subjects learn to associate nonsense-syllable CVCs with four circles of differing sizes. Half of the subjects learned circles that ranged from 12 to $18 \mathrm{~mm}$ in diameter, whereas the others learned circles that ranged from 12 to $24 \mathrm{~mm}^{2}$ The subjects were then presented with CVC pairs and were asked to choose the CVC associated with the larger circle. In addition to the usual symbolic distance effect, it was found that for each ordinal distance between pairs, subjects who had learned circles with the larger interval distances between pairs responded faster. Furthermore, the pattern of data for these two groups closely paralleled the pattern found for subjects in two control groups who performed perceptual comparisons on the same stimuli. Thus, it was concluded that absolute size information was represented in memory, that this information played a functional role in the performance of this task, and that the processes in the symbolic comparisons were similar if not identical to those involved in the perceptual comparisons.

Although there have been other claims of support for analogue processing in symbolic comparison tasks (e.g., Holyoak \& Walker, 1976; Kosslyn et al,, 1977; McKinley-Brewer, 1979; Paivio, 1975), the range effect demonstrated by Moyer and Bayer (1976) provides the most compelling evidence. The finding that absolute differences in size of the referents affect symbolic comparison time is especially strong evidence that the memorial representation varies directly with the physical stimulus as an analogue model requires, and runs counter to the assumption of the semantic coding model that tags are assigned on the basis of ordinal position. Furthermore, the fact that these results are found in a task that repeatedly compares pairs of the same four symbols argues that analogue processes operate even under conditions in which they do not seem necessary

It is important to note that only the Moyer and Bayer (1976) study has documented the range effect and that a recent attempt by Banks, Mermelstein, and Yu (1982) to find a range effect in a symbolic comparison task was unsuccessful. Since the Moyer and Bayer result is so provocative and so widely cited (Banks et al., 1982, noted 26 different articles that cite Moyer and Bayer by the spring of 1981), it seems worthwhile to try to discover why Banks et al. were unable to replicate it.

There are several possible explanations for the inability of Banks et al. (1982) to obtain a range effect. One possibility is that subjects in the Banks et al. study may have been allowed to view groups of stimuli when initially learning the symbolic associations. Allowing subjects to see more than one circle at a time could have encouraged them to encode the circles in terms of their relative, rather than their absolute, sizes. If it is true that analogue processing occurs in symbolic comparisons only when information about relative positions along a dimension has not been perceptually detected and prestored, then the range effect would be expected to disappear under conditions in which all stimuli appeared together.

A second difference between the two studies is the use of practice trials. Whereas Moyer and Bayer (1976) specifically reported that they gave no practice trials, Banks et al. (1982) gave practice trials in three of their four experiments, and in the one experiment without practice trials (Experiment 2), the data were not analyzed by block. It is possible that subjects in this task first use an analogue comparison process but then generate discrete codes once the experimental situation becomes clear. If this is true, any analogue processing performed would occur during the practice trials or the earliest test trials.

Third, in their crucial Experiment 2, Banks et al. (1982) compared the functions relating actual and remembered stimulus sizes and found that the subjects in their smallrange group were remembering relatively greater distance per actual unit distance than were the subjects in the medium-range condition, who in turn remembered greater distance per actual unit distance than did the subjects in the large-range condition (see Banks et al., 1982, Figure 3). Since encoded distance is the variable of interest, these differences in encoding may be problematic.

Fourth, although the distance effect is not an artifact of end-term processing (Holyoak \& Walker, 1976; Potts, 1974; Scholz \& Potts, 1974), it has been shown that different strategies may be used depending on whether or not a given pair contains an end term (Kosslyn et al., 1977; McKinley, 1975; Potts, 1974). For example, Potts (1974) presented evidence suggesting that the comparison process may be bypassed entirely if the first member of the comparison pair is an end term. In studies looking for a range effect, it is possible that end-term processing is equivalent for both the large-range and small-range groups, making it difficult to detect a range effect occurring in comparisons not containing an end term.

The present study was an attempt to find a range effect in the light of the concerns expressed above. Two groups of subjects compared symbolic stimuli that had as referents sets of six circles, with each set differing in the interval distance between pairs. Care was taken not to en- 
courage the encoding of relative, as opposed to absolute, size information, and there were no practice comparison trials. Since subjects may have employed a different strategy when dealing with end-term comparisons, stimulus sets contained six members and data were analyzed both with and without end-term trials. Also, if comparisons are based solely on ordinal information, judgments should be similar for stimuli that have circles of differing sizes as referents and stimuli that do not have absolute size referents at all. Accordingly, a third group of subjects was added that made size comparisons among symbolic stimuli for which only an arbitrary linear ordering had been learned.

\section{Method}

\section{Subjects}

Thirty-six University of Massachusetts undergraduates participated in this experiment in return for credit toward course grades.

\section{Apparatus}

Stimulus letters were presented on a CRT controlled by a HewlettPackard 2114B computer. The letters were presented in the center of the screen, in uppercase, each approximately $50 \mathrm{~mm}$ tall and $20 \mathrm{~mm}$ wide, separated by approximately $40 \mathrm{~mm}$ of blank space. Response choices and reaction times (RTs) were obtained via a microswitch console and were recorded by the computer.

\section{Materials \\ Two sets of six circles each were drawn on $7.6 \times 12.7 \mathrm{~cm}$ in- dex cards using an ultrafine $(0.3-\mathrm{mm})$-tipped black felt lettering pen and a professional engineering template. One circle was drawn in the center of each card. One set of six cards (large range, LR) had circles with diameters of $8,12,16,20,24$, and $28 \mathrm{~mm}$, whereas the other set (small range, SR) had circles with diameters of 10 , $12,14,16,18$, and $20 \mathrm{~mm}$. The middle four circles of each range were the same sizes as those used by Moyer and Bayer (1976). \\ Six common color names (Yellow, Orange, Black, Red, Green, and White) differing in their first letters were chosen to serve as the symbolic associates of the circles. Color names were assigned to circles according to a Latin square, such that all circle-color pairs occurred equally often across subjects and each color appeared equally often at each ordinal position. ${ }^{3}$}

\section{Procedure}

Each subject was tested individually in a session consisting of a learning phase, a test phase, and an assessment phase. Twelve subjects were assigned randomly to each of the experimental conditions, and were assigned randomly to one of the six circle-color pairings within each group (or, in the case of the colors-only group, to one of the six color orderings).

Learning phase. The subjects in the LR and SR groups were taught the circle-color associations by means of a procedure designed to ensure an equivalent degree of learning for each subject both within and between groups.

First, each subject was given a general overview of this phase. The overview emphasized the difficulty of the associations to be learned and directed the subject's attention to the absolute sizes of the circles. The subject was further informed that $s /$ he would later be tested on how well the circle-color pairs had been learned. The relative judgment was not mentioned at this time.

Each subject was then shown each of the six circles in the assigned deck, one at a time, in the following manner: The card containing a circle was placed on the table in front of the subject, approximately $30 \mathrm{~cm}$ from the subject's eyes. The experimenter then spoke aloud the color name. The subject was allowed to study the circle as long as $\mathrm{s} / \mathrm{he}$ wished. When the subject was ready, the card was removed and a new card was presented, along with its color name. When all six cards had been presented, the deck was shuffled and presented again, in the same manner, until the deck had been presented five times.

Next, each subject was again shown the six circles, one at a time, but now was asked to provide the appropriate color. Wrong answers were corrected by the experimenter, and the subject was again allowed to study each circle as long as s/he wished. When the subject had correctly named the entire deck four consecutive times, the deck was replaced by a duplicate deck (to guard against strategies based on abnormalities in the cards). This duplicate deck was then presented in the same manner until the entire deck had been correctly named once.

The subjects in the colors-only (CO) group, unlike those in the LR and SR groups, were taught the linear ordering of the colors in a manner that made it clear that the ordering was totally arbitrary. These subjects were told that the colors would form an ordering, and that a test would later be given on how well the ordering had been learned. Again, the exact nature of the later test was not mentioned at this time. The experimenter verbally presented each of the five color pairs, consisting of adjacent colors in the ordering, along with the relation between them. For example, if the ordering to be learned was Yellow-Orange-Black-Red-Green-White, with left representing small and right large, then half of the subjects in the $\mathrm{CO}$ group were taught "Yellow is smaller than Orange; Orange is smaller than Black; Black is smaller than Red," etc., whereas the other half of the subjects were taught the ordering from the opposite end, as "White is larger than Green; Green is larger than Red; Red is larger than Black," etc. Note that the color pairs were always presented from end to end, as the examples illustrate. The experimenter ran through the ordering in this manner five times.

Each subject in the CO group was then presented with the six colors in a question format and was asked to give the next smallest or next largest color, with the question taking the same form as had the presentation. For example, the subjects who had learned the above ordering from the small end would be asked "Yellow is smaller than _.?" and would be required to provide the next immediate color, in this case "Orange." (Note that the correct response to "White is smaller than _.?" in this example would be "nothing.") The six questions comprising the six colors were asked in blocks (each question being asked once per block), with the order of questions random within blocks. The questions were presented in this manner until each question had been answered correctly five consecutive times.

Several points about the learning phase warrant emphasis. First, as stressed above, none of the subjects in any group knew that they would be tested in a relative judgment task. Second, only subjects in the CO group were specifically told to learn the stimuli as a relative linear ordering. Third, each member of any one group had a counterpart in the other two groups who learned the same ordinal correspondence between colors. This served to ensure that any order effects associated with the colors would be controlled through occurrence in all groups. Finally, the subjects in the LR and SR groups never had more than one circle in view at any one moment.

Test phase. The subjects sat in a dimly lit, sound-dampened room, with their eyes approximately $65 \mathrm{~cm}$ from the CRT and with their hands resting comfortably on the response console. The experimenter explained the procedure, answered questions, and left the room.

Test trials were organized into six blocks per subject. No practice trials were given. A block was initiated by the subject, who began the block by depressing the microswitch at any time after the computer had displayed a ready signal on the CRT. There was then $1 \mathrm{sec}$ of blank screen between offset of the ready signal and the beginning of the first trial of that block.

Each block consisted of the 15 possible pairs of the six colors, once in each of the two possible orders, for a total of 30 trials per block. The presentation order of the 30 trials within each block was randomized. There was a mandatory rest period of about $20 \mathrm{sec}$ 
between blocks, after which the ready signal reappeared, allowing the subject to initiate the next block.

Each trial consisted of the display of a fixation cross for $500 \mathrm{msec}$, a blank screen for $500 \mathrm{msec}$, and then onset of the first letters of two of the six learned color names (e.g., "R G"). The two letters remained on the screen until the subject had responded by pushing the microswitch on the same side as the letter (standing for the color) that was associated with the larger circle (or simply the larger color in the case of the $\mathrm{CO}$ group). The letters disappeared with the response, followed by $500 \mathrm{msec}$ of blank screen and then the fixation cross for the next trial.

The subjects were instructed to respond as fast as possible to each comparison but to make as few errors as possible. No feedback was given on either errors or speed of response at any time during the test phase.

Assessment phase. Following the test phase, the subjects' memories of the absolute sizes of the circles were assessed. The subjects in the LR and SR groups were first asked to draw the circles as accurately as they could from memory. A randomly selected color was named, and a subject drew the circle corresponding to that color on a slip of paper that was the same size as the index cards upon which the circles had originally appeared. When the subject had completed the drawing, the paper was removed from sight and the next color was named. This was continued until all circles had been drawn. Note that the subjects were not allowed to use their previously drawn circles as visual references from which to draw subsequent circles.

Next, subjects in the LR and SR groups were asked to estimate the diameter of each circle, in order, from smallest to largest, by placing hashmarks on a set of horizontal lines provided for this purpose. In this case, the subjects were allowed to see all of their estimates simultaneously.

Finally, the subjects from all three groups were asked to describe any strategies that they had used during the relative judgment task.

\section{RESULTS}

RT data were subjected to analyses of variance that included the between-subjects factor of group (LR, SR, or $\mathrm{CO}$ ) and the within-subjects factors block (six levels) and ordinal distance ${ }^{4}$ (three or five levels, depending on whether comparisons involving end terms were included or not). The data are displayed in Figure 1.

\section{Analyses Including End-Term Comparisons}

Contrary to the analogue comparison hypothesis, there was no tendency for mean RT to differ among the SR, LR, and CO groups $[F(2,33)<1]$. In fact, mean RT was slightly greater for the LR group than for the SR group, both overall and for the first few blocks, although these differences did not approach significance $(\mathrm{Fs}<1)$. For all three groups, RT decreased as the distance between members of comparison pairs increased. Overall, the main effect of distance was highly significant $[F(4,132)=$ $125.7, \mathrm{p}<.001 \mathrm{l}$, and there was no suggestion of an interaction between distance and group $(F<1)$. RT decreased with practice, resulting in a significant block main effect $[F(5,165)=57.9, p<.001]$. Collapsing over distance and group, the mean RTs for the six blocks were $1,492,1,032,964,912,848$, and $836 \mathrm{msec}$, respectively. Both the group $\times$ block $[\mathrm{F}(10,165)=1.74]$ and the distance $\times$ block $[\mathrm{F}(20,660)=1.52]$ interactions approached significance $(.05<\mathrm{p}<.10)$. In the former case, there

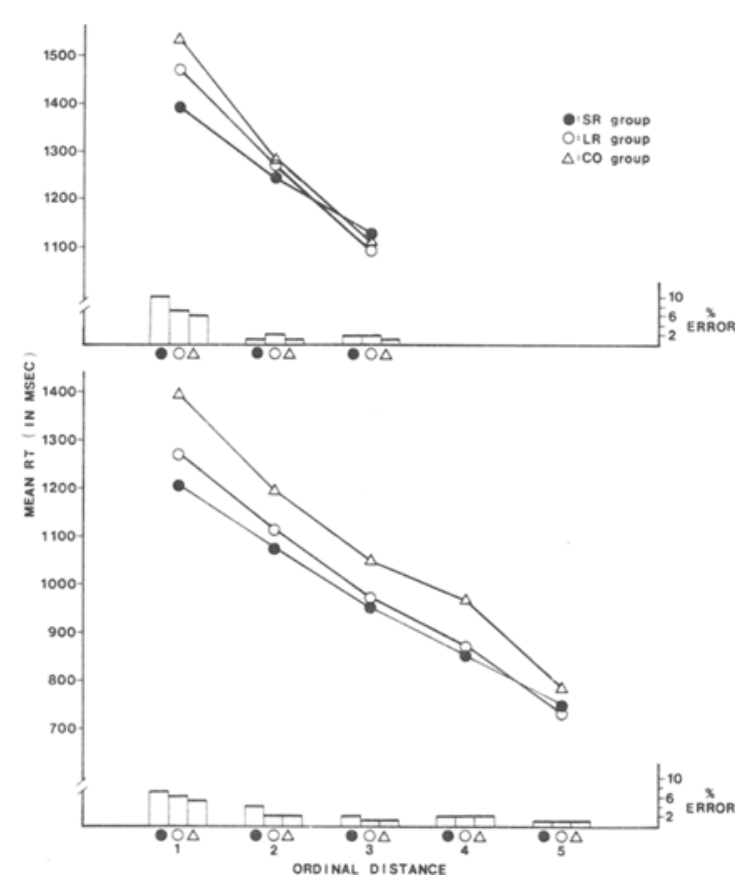

Figure 1. Mean RT and percent error as a function of ordinal distance for the small-range (SR), large-range (LR), and colors-only (CO) groups with the end terms excluded (upper panel) and included (lower panel).

was no suggestion of an interaction when the $\mathrm{CO}$ group was removed from the analysis $[F(5,110)<1]$, and hence no support was lent to the notion of a range effect that diminished rapidly with practice. In the latter case, there was a tendency for comparisons involving the end terms both to be faster and to improve less with practice than the other comparisons. There was no suggestion of a distance $\times$ block interaction $[F(10,330)<1]$ when comparisons involving end terms were removed from the analysis.

\section{Analyses Excluding End-Term Comparisons}

When comparisons involving end terms were excluded, the results closely paralleled those reported above. There was no effect of group whether or not the $\mathrm{CO}$ condition was included in the analysis (Fs $<1$ ). Again, there was a tendency for the LR comparisons to take longer, both overall and in the first few blocks, although the differences did not approach significance. The effect of ordinal distance was significant $[F(2,66)=63.5, p<.001]$ and did not interact with group $[\mathrm{F}(4,66)=1.06, \mathrm{p}>$ $.25]$. There was a significant effect of block $[F(5,165)$ $=56.9, \mathrm{p}<.001]$, and an interaction between group and block $[\mathrm{F}(10,165)=2.16, \mathrm{p}<.05]$ that disappeared when the $\mathrm{CO}$ group was removed from the analysis $[F(5,110)=1.67, p>.10]$. The reason for the significant interaction when the $\mathrm{CO}$ group was included seems to be that performance in the LR and SR groups tended to level off after the fifth block, whereas the $\mathrm{CO}$ group continued to improve. 
Table 1

Mean Reaction Time (in Milliseconds) Adjusted for Error Rate and Trials to Criterion

\begin{tabular}{|c|c|c|c|c|c|c|c|}
\hline \multirow[b]{2}{*}{ Group } & \multicolumn{5}{|c|}{ Ordinal Distance } & \multirow{2}{*}{$\begin{array}{c}\text { Unweighted } \\
\text { Mean }\end{array}$} & \multirow{2}{*}{$\begin{array}{c}\text { Weighted* } \\
\text { Mean }\end{array}$} \\
\hline & 1 & 2 & 3 & 4 & 5 & & \\
\hline \multicolumn{8}{|c|}{ With End Terms } \\
\hline LR & 1253 & 1112 & 973 & 869 & 742 & 990 & 1074 \\
\hline SR & 1226 & 1087 & 955 & 844 & 759 & 974 & 1053 \\
\hline \multicolumn{8}{|c|}{ Without End Terms } \\
\hline LR & 1483 & 1272 & 1089 & & & 1281 & 1347 \\
\hline SR & 1443 & 1276 & 1142 & & & 1287 & 1337 \\
\hline
\end{tabular}

Note $-L R=$ large range; $S R=$ small range. ${ }^{*}$ The weighted mean took account of the number of comparisons at each ordinal distance.

\section{Additional Analyses}

Two additional aspects of the data should be noted: (1) the SR group required more trials on the average to reach criterion than did the LR group (16.3 vs. 13.2), and there was a slight negative correlation $(r=-.17)$ between trials to criterion and RT during the test phase of the study, and (2) the SR group had a slightly higher error rate than the LR group, both for comparisons involving end terms $(4.7 \%$ vs. $3.7 \%)$ and comparisons not involving end terms (5.7\% vs. $4.6 \%)$. Although none of these differences were significant $(p>.20$ for trials to criterion; $\mathrm{p}>.10$ for the correlation; and $\mathrm{ps}>.25$ for the error rates), to the extent that such differences may have been present, they would operate to decrease RT of the SR group relative to that of the LR group. Consequently, RT was adjusted for the difference in error rate using the correction (Yellot, 1971) used by Moyer and Bayer (1976), and a regression correction was used to adjust for the difference in the number of trials to criterion between the LR and SR groups. (For example, regressing RT for comparisons with end terms included on the number of trials to criterion in the learning phase, the 3.2trial difference between the SR and LR groups corresponds to $12 \mathrm{msec}$.) Applying both corrections served merely to bring the LR and SR groups closer together. The adjusted means are given Table 1 .

There is no reason to think that RT showed only ordinal effects because of a failure to retain absolute size information. In the assessment phase (which followed the test phase), the subjects were able to draw the circles very accurately. For each circle drawn, the estimated diameter was defined as the average of three diameters measured at $120^{\circ}$ intervals. Figure 2 displays estimated diameter as a function of actual diameter. Although Figure 2 shows some underestimation of circle size, interval information was well retained. The estimated diameters of ordinally adjacent circles in the LR condition differed on average by $4.7 \mathrm{~mm}$, as compared with the actual difference of $4.0 \mathrm{~mm}$. The corresponding figures for the SR condition were 2.4 and $2.0 \mathrm{~mm}$. In a second estimation task, the subjects indicated diameter length by placing marks on a line. The mean differences between the estimated lengths of the diameters of the largest and smallest circles were $22.0 \mathrm{~mm}$ in the LR condition and $10.6 \mathrm{~mm}$ in the SR condition. The actual differences were 20 and $10 \mathrm{~mm}$.

\section{DISCUSSION}

The failure to find a significant difference between the groups that learned different interval distances between circles calls into question the results reported by Moyer and Bayer (1976). In the present study, not only was the difference between the LR and SR groups not significant, it was not in the predicted direction. In addition, there was no indication that interval distance affected early trials but not later trials, as would be the case if absolute size information were used to make comparisons only until a discrete data base could be constructed. The lack of an effect also cannot be accounted for by different memory functions produced by subjects in the two circle-learning groups (see Figure 2), as may have been the case in Banks et al. (1982, Figure 3). Furthermore, the high degree of similarity in the present study among the comparison time functions produced by the two groups that learned absolute size referents for the compared symbols and the group that learned only the symbols in an arbitrary linear ordering strongly suggests that the same comparison process was used in both cases.

At this point, we cannot explain the difference between Moyer and Bayer's (1976) findings and our own. Without a replicable range effect, however, the single most direct source of support for the use of absolute size information in symbolic comparisons is called into question, since the Moyer and Bayer study was a direct attempt to build up a known analogue data base in memory. And without a clear demonstration that absolute size information is of functional consequence in the relative judgment task, the models of symbolic comparison that are based upon the processing of analogue representations are seriously undermined, because they all predict that absolute size in-

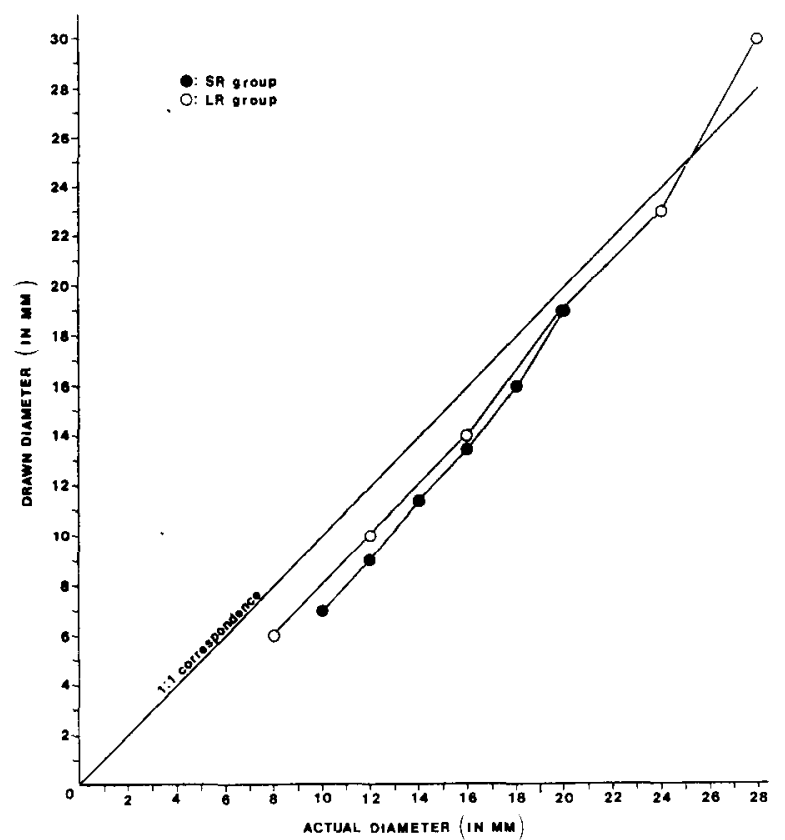

Figure 2. Mean diameters of circles drawn in the assessment phase as a function of actual circle size. 
formation will affect comparison time. If, as suggested by the present study, comparison time is not affected by the absolute distance between physical referents, and comparisons are made in a virtually identical manner whether or not the symbols refer to actual physical objects, then there seems to be no reason to prefer the analogue model over a model that posits that abstract information is used to make a decision.

The study reported here, following the studies of Moyer and Bayer and Banks and his colleagues, can be described as involving a finite (indeed, small) set of arbitrarily ordered symbols. As Banks (1977) pointed out, in tasks such as this, it is quite easy for subjects to keep in mind the entire set of symbols correctly ordered on the proper dimension. Introspective reports obtained from the subjects in the present experiment agree with this conclusion. Virtually all the subjects reported that their strategy involved some combination of using the end terms when they were present in the pair in order to make a quick decision (as can clearly by seen in Table 1) and simply using the linear ordering of the colors otherwise. Even when explicitly asked whether they had ever pictured the circles to make a decision (an obviously leading question), most of the subjects said that they had not because there was no need to do so. Since the number of items in these studies never exceeded the commonly cited capacity of short-term memory (Miller, 1956), the entire ordered set could be maintained in an activated state.

An analogue comparison process would be much more useful if it were difficult to generate or maintain an explicit ordering of items (so that information would have to be drawn from semantic memory), as would be the case if large sets of items were presented and a given item were not involved in many comparisons (see, e.g., Paivio, 1975). Still, it should be noted that several studies have shown interval scale effects above and beyond ordinal scale effects in repeated-set paradigms, both with preexperimentally (e.g., Banks \& Flora, 1977; Friedman, 1978) and arbitrarily (e.g., Griggs \& Shea, 1977) defined orderings, and even after many comparison trials with the same stimuli (Banks \& White, 1982). The problem with these studies is that none of them directly implicated analogue processes.

It may be argued that the finite, arbitrarily ordered stimulus set paradigm is in fact the most artificial, least ecologically valid form of the task. Generally, when people make symbolic comparisons of physical objects in the course of normal cognitive functioning ("Is your car bigger than mine?"), the symbols (i.e., words) compared are members of a virtually infinite set, the set is not maintained as a linear ordering, and the symbols are semantically related to the physical items that they represent via the lexicon. Although the failure to obtain a range effect in the present study in conjunction with the Banks et al. (1982) study is inconsistent with the use of an analogue comparison process when small sets of nonsemantically related symbols are used, this does not preclude the possibility that analogue comparison processes may be used in other symbolic comparison situations. The problem, now, is to devise an experimental paradigm that provides a means for determining whether the effects of absolute size found in other versions of the symbolic comparison task are in fact produced by an analogue comparison process.

\section{REFERENCES}

BANKS. W. P. (1977). Encoding and processing of symbolic informa tion in comparative judgements. In G. H. Bower (Ed.), The psychology of learning and motivation (Vol. 11). New York: Academic Press

Banks, W. P. \& Flora, J. (1977). Semantic and perceptual processes in symbolic comparison. Journal of Experimental Psychology: $\mathrm{Hu}$ man Perception and Performance, 3, 278-290.

Banks, W. P., Mermelstein, R., \& YU, H. K. (1982). Discriminations among perceptual and symbolic stimuli. Memory \& Cognition, 10, 265-278.

BANKS, W. P., W WITE, H. (1982). Single ordering as a processing limitation. Joumal of Verbal Learning and Verbal Behavior, 21, 39-54

Banks, W. P., White, H., Sturgill, W., \& Mermelstein, R. (1983). Semantic congruity and expectancy in symbolic judgments. Journal of Experimental Psychology: Human Perception and Performance, 9, $560-582$

Bucklfy, P. B., \& Gillman, C. B. (1974). Comparisons of digits and dot patterns. Journal of Experimental Psychology, 103, 1131-1136.

FINKE, R. A. (1980). Levels of equivalence in imagery and perception Psychological Review, 87, 113-132.

FriEdmAN, A. (1978). Memorial comparisons without the "mind's eye." Journal of Verbal Learning and Verbal Behavior, 17, 427-444.

GrigGs, R. A., \& SHEA, S. Z. (1977). Integrating verbal quantitative information in linear orderings. Memory \& Cognition, 5, 287-291.

Holyoak, K. J., Dumais, S. T., \& Moyer, R. S. (1979). Semantic association effects in a mental comparison task. Memory \& Cogni tion, 7, 303-313

Holyoak, K. J., Walker, J. H. (1976). Subjective magnitude information in semantic orderings. Journal of Verbal Leaming and Ver bal Behavior, 15, 287-299

Jamieson, D. G., \& Petrusic, W. M. (1975). Relational judgments with remembered stimuli. Perception \& Psychophysics, 18, 373-378.

Kosslyn, S. M., Murphy, G. L., Bemesderfer, M. E., \& Feinstein, K. J. (1977). Category and continuum in mental comparisons. Journal of Experimental Psychology: General, 106, 341-375.

MARKS, D. F. (1972). Relative judgment: A phenomenon and a the ory. Perception \& Psychophysics, 11, 156-160.

MCKINLEY. J. E. (1975). Utilizing comparative information from memory: The symbolic distance effect. Unpublished master's thesis University of Massachusetts, Amherst.

MCKINLEY-BREWER, J. E. (1979). The representation of relational in formation in long term memory. Unpublished doctoral dissertation. University of Massachusetts, Amherst.

Miller, G. A. (1956). The magical number seven, plus or minus two: Some limits on our capacity for processing information. Psychological Review, 63, 81-97

Moyer, R. S. (1973). Comparing objects in memory: Evidence sug gesting an internal psychophysics. Perception \& Psychophysics, 13. 180-184.

Moyer, R. S. \& BAyer, R. H. (1976). Mental comparison and the symbolic distance effect. Cognitive Psychology, 8, 228-246.

Moyer, R. S., \&umais, S. T. (1978). Mental comparison. In G. H. Bower (Ed.), The psychology of learning and motivation ( $\mathrm{Vol}$. 12). New York: Academic Press.

PaIvio, A. (1975). Perceptual comparisons through the mind's eye. Memory \& Cognition, 3, 635-647.

PAtvio, A. (1978). Mental comparisons involving abstract attributes. Memory \& Cognition. 6, 199-208.

Porrs, G. R. (1972). Information processing strategies used in the encoding of linear orderings. Journal of Verbal Learning and Verbal Behavior. 11, 727-740. 
PotTs, G. R. (1974). Storing and retrieving information about ordered relationships. Journal of Experimental Psychology, 103, 431-439.

Potts, G., Banks, W. P., Kosslyn, S. M., Moyer, R. S., Riley, C., \& SMITH, K. (1978). Encoding and retrieving ordered relationships. In N. J. Castellan \& F. Restle (Eds.), Cognitive theory (Vol. 3) Hillsdale, NJ: Erlbaum.

Scholz, K. W., \& PotTs, G. R. (1974). Cognitive processing of linear orderings. Journal of Experimental Psychology, 102, 323-326.

ShePard, R. N., \& Podgorny, P. (1978). Cognitive processes that resemble perceptual processes. In W. K. Estes (Ed.), Handbook of learning and cognitive processes (Vol. 5). Hillsdale, NJ: Erlbaum.

Woocher, F. D., Glass, A. L., \& Holyoak, K. J. (1978). Positional discriminability in linear orderings. Memory \& Cognition, 6, 165-173.

YelLot, J. I., JR. (1971). Correction for fast guessing and the speedaccuracy tradeoff in choice reaction time. Journal of Mathematical Psychology, 8, 159-199.

\section{NOTES}

1. The term "symbolic comparison" is something of a misnomer, since it is not the symbols that are compared. "Symbolic referent com- parison" would be a more appropriate term. However, in staying with convention, we will use the former.

2. Whereas Moyer and Bayer (1976) reported that the stimuli were 11 to $17 \mathrm{~mm}$ and 11 to $23 \mathrm{~mm}$ for the two groups, a recent paper by Banks, Mermelstein, and Yu (1982) reported that the Moyer and Bayer stimuli were each $.8 \mathrm{~mm}$ larger than originally reported, and therefore the sizes have been rounded up in this study. It should be noted that the interval distances between the circles remain the same.

3. Color names were used because pilot work had indicated that subjects had difficulty learning six CVCs at the same time that they learned six difficult associations. The color names were randomly ordered before they were entered into the Latin square. In addition, analyses of the experimental data did not suggest any systematic effect of color name on RT.

4. If one ranks the stimuli in terms of size, the ordinal distance between two stimuli corresponds to the difference between their ranks. For example, with a stimulus set containing six circles of monotonically increasing size, the distance between the largest and smallest stimuli would be 5 .

(Manuscript received October 17, 1984 revision accepted for publication March 15, 1985.) 